\title{
Recent developments in the effort to cure HIV infection: going beyond $\mathbf{N}=1$
}

\author{
Janet D. Siliciano' ${ }^{1}$ and Robert F. Siliciano ${ }^{1,2}$ \\ "Johns Hopkins University School of Medicine, Baltimore, Maryland, USA. ${ }^{2}$ Howard Hughes Medical Institute, Baltimore, Maryland, USA.
}

\begin{abstract}
Combination antiretroviral therapy (ART) can suppress plasma HIV to undetectable levels, allowing HIV-infected individuals who are treated early a nearly normal life span. Despite the clear ability of ART to prevent morbidity and mortality, it is not curative. Even in individuals who have full suppression of viral replication on ART, there are resting memory $\mathrm{CD4}^{+} \mathrm{T}$ cells that harbor stably integrated HIV genomes, which are capable of producing infectious virus upon T cell activation. This latent viral reservoir is considered the primary obstacle to the development of an HIV cure, and recent efforts in multiple areas of HIV research have been brought to bear on the development of strategies to eradicate or develop a functional cure for HIV. Reviews in this series detail progress in our understanding of the molecular and cellular mechanisms of viral latency, efforts to accurately assess the size and composition of the latent reservoir, the characterization and development of HIV-targeted broadly neutralizing antibodies and cytolytic T lymphocytes, and animal models for the study HIV latency and therapeutic strategies.
\end{abstract}

The past few years have seen a remarkable process of coalescence in which several distinct areas of AIDS research have been brought to bear on the search for a cure. The development of effective combination antiretroviral therapy (ART) in the mid-1990s initially raised hopes for a cure because plasma levels of HIV fell quickly to undetectable levels in treated patients (1-3). However, simultaneous studies demonstrated the presence in infected individuals of resting memory $\mathrm{CD} 4^{+} \mathrm{T}$ cells harboring stably integrated viral genomes that did not produce infectious virus while the cells were in a resting state but could do so following $\mathrm{T}$ cell activation $(4,5)$. With the subsequent demonstration that this latent reservoir could persist indefinitely, even in the setting of optimal ART (6-11), hopes for a cure faded rapidly. In fact, cure became an almost taboo subject because of concerns that discussing cure would raise unrealistic expectations in infected individuals that this reservoir could ever be eliminated. In the meantime, steady improvements were made in the tolerability and convenience of ART regimens, such that infected individuals can now maintain indefinite suppression of viral replication to clinically undetectable levels on single-pill, once-daily regimens that have few side effects (12). Recent studies suggest that infected individuals starting treatment early with modern regimens have near-normal life expectancies (13-16).

The success of ART is one reason that there has been renewed interest in HIV cure. In addition, recent studies, beginning with the pioneering work of David Margolis and colleagues, have indicated that the latent reservoir can be perturbed in vivo with latencyreversing agents (LRAs) $(17,18)$. Importantly, the revival of optimism for HIV cure was further stimulated by the remarkable case

Conflict of interest: The authors have declared that no conflict of interest exists. Reference information: J Clin Invest. 2016;126(2):409-414. doi:10.1172/JCI86047. of Timothy Brown, a courageous patient with HIV infection who received a hematopoietic stem cell transplant (HSCT) for leukemia from a carefully selected donor whose cells were resistant to HIV infection $(19,20)$. To date, Mr. Brown is the one and only person considered to be cured. His case gave rise to renewed hope that the problems posed by the latent reservoir could be overcome. In this issue of the JCI, a distinguished group of experts review exciting recent developments in the HIV cure field and describe how research discoveries in the areas of HIV pathogenesis, vaccines, and treatment are all now contributing to the search for a cure.

\section{The latent reservoir as a barrier to cure}

Although other reservoirs for HIV may exist, the latent reservoir in resting $\mathrm{CD}^{+} \mathrm{T}$ cells is considered to be the major barrier to curing HIV infection. In everyone with HIV infection, regardless of how long they have been on suppressive ART, replication-competent virus can be isolated from resting $\mathrm{CD} 4^{+} \mathrm{T}$ cells simply by activating the cells in vitro (6-10). T cell activation serves to reverse the state of latency that is observed in resting $\mathrm{CD} 4^{+} \mathrm{T}$ cells, allowing the outgrowth of virus $(4,5)$. This concept underlies the quantitative viral outgrowth assay (QVOA) that is considered to be the "gold-standard" method for measuring the latent reservoir. The important problem of measuring the latent reservoir is discussed in detail by Marta Massanella and Douglas Richman in this issue (21). Longitudinal measurements by the QVOA in patients on suppressive ART established that the half-life of the pool of latently infected resting $\mathrm{CD}^{+} \mathrm{T}$ cells is extremely long (3.7 years), thus requiring over 70 years of treatment to eradicate a pool of $10^{6}$ cells $(9,10)$. This estimate was based on studies published in 1999 and 2003, when ART regimens still had considerable problems with side effects. Recently, Margolis and colleagues published a new longitudinal analysis of the half-life of the latent reservoir, as measured by the QVOA (22). Although many of the patients in this 
study were on newer, less toxic regimens, the observed half-life was essentially the same (3.6 years). This means that the improvements in ART over the last several years have not affected the fundamental problem of HIV persistence in latently infected cells. The persistence of replication-competent HIV on a time scale of years in the setting of optimal ART has not been demonstrated for any other cell population, and thus the latent reservoir in resting $\mathrm{CD} 4^{+} \mathrm{T}$ cells is a major focus of HIV cure research.

The question of why HIV establishes a state of latent infection is of considerable interest. Latency is a reversibly nonproductive state of infection of individual cells. For some viruses, especially those of the herpes virus family, latency is an important mechanism for immune evasion (23). It is not so clear that latency serves this function for HIV. Pioneering studies by Jeff Lifson and colleagues established that HIV replicates actively throughout the course of the infection in untreated individuals (24). The rapid evolution of escape mutants provides the principal mechanism by which the virus avoids elimination by antibody and cytolytic T lymphocyte (CTL) responses (25-30). Intriguing recent theoretical studies by Leor Weinberger suggest that latency is a "hard-wired" feature of the regulation of HIV gene expression that evolved as a "bet-hedging strategy" to allow the virus to be successfully transmitted across mucosal barriers, with subsequent reactivation once the initially infected cells reach a tissue site that is more favorable for viral replication $(31,32)$. If this hypothesis is correct, the form of latency involved is likely different from the one that allows persistence of the virus during ART. As is discussed below, the latter form of latency allows infected cells to persist for very long periods of time (months to years) without reactivation. In contrast, a long delay between mucosal exposure and systemic viral replication has never been reported.

The simplest explanation for the existence of the latent reservoir is that HIV latency is a consequence of viral tropism for activated $\mathrm{CD} 4^{+} \mathrm{T}$ cells (4). T cell activation results in a gradual upregulation of the CCR5 coreceptor that is essential for entry of the commonly transmitted forms of HIV (33). Activation also increases deoxynucleoside triphosphate (dNTP) pools required for reverse transcription and releases sequestered forms of the host transcription factors NF- $\kappa B$, nuclear factor of activated T cells (NFAT), and positive transcription elongation factor-b (PTEFb), all of which play an important role in HIV gene expression (as reviewed by Daniele Cary, Koh Fujinaga, and B. Matija Peterlin in this issue; ref. 34). Infection of activated $\mathrm{CD} 4^{+} \mathrm{T}$ cells results in rapid reverse transcription, integration, viral gene expression, and virus production, followed generally by the death of the cells, usually in one to two days $(35,36)$. In contrast, infection of resting $\mathrm{CD}^{+} \mathrm{T}$ cells is hindered by the absence of CCR5, by low levels of dNTPs maintained by the dNTP triphosphohydrolase SAMHD1 $(37,38)$, and by a recently described cell death pathway that is triggered by innate immune recognition of reverse transcription intermediates (39). While infection of both activated and resting $\mathrm{CD} 4^{+} \mathrm{T}$ cells results in cell death, infection of activated $\mathrm{CD} 4^{+} \mathrm{T}$ cells that are transitioning back to a resting state, while the cells are still permissive for reverse transcription and integration of the viral genome but not for high-level viral gene expression, may allow the establishment of a stable state of latent infection in resting $\mathrm{CD} 4^{+} \mathrm{T}$ cells (4). This is a rare event, consistent with the extremely low frequency of latently infected cells in vivo $\left(1: 10^{6}\right)$. Viewed in this light, HIV latency is an unfortunate accident of viral tropism. Whether this or other explanations for the origins of HIV latency are correct, there is no doubt that latency is established in all infected individuals and that it serves as a formidable barrier to HIV cure.

\section{A cure and some "near cure" cases}

The single cure of HIV infection mentioned above involved a HSCT from an HLA-matched donor selected to be homozygous for a common 32-base pair deletion in the CCR5 gene (19). As is reviewed by Daniel Kuritzkes in this issue (40), elimination of the latent reservoir in this patient likely involved the conditioning regimen given prior to transplant as well as the graft-versus-host reactions that occurred following transplant. Together, these factors resulted in complete or near-complete replacement of the host immune system (including latently infected cells) with HIV-resistant donor cells. Even if a small number of infected cells remained in this patient, any virus released would not be able to infect the HIV-resistant, donor-derived cells that now constitute his immune system. This remarkable case has proven that cure is possible.

Even more instructive were two subsequent cases in which HIV-infected individuals with recurrent lymphomas were given HSCTs from donors who were wild type at the CCR5 locus (41, 42). ART was continued throughout the transplant period and for several years thereafter to protect donor cells from infection. ART was then discontinued, and, in both cases, the plasma virus levels remained below the limit of detection for several months. Normally there is an exponential increase in viremia at approximately two weeks after treatment interruption $(43,44)$. Both patients eventually experienced a sudden and dramatic rebound in viremia (at 3 and 8 months after ART interruption), presumably as the result of reactivation of one or more residual latently infected cells. A similar delay in viral rebound was observed in the "Mississippi baby," an infant born to an infected mother who had no prenatal care (45). High levels of HIV were detected in the baby shortly after birth, and an aggressive ART regimen was started immediately. Viremia fell rapidly to below the limit of detection and remained undetectable even after ART was interrupted (against medical advice) at approximately 15 months. Initially, there was hope that early ART had prevented the establishment of the latent reservoir, since the reservoir resides in memory $\mathrm{CD} 4^{+} \mathrm{T}$ cells that are largely generated by exposure to antigen in postnatal life. However, there was again a sudden and dramatic rebound in viremia over 2 years after interruption of ART. Interestingly, all three cases were characterized by a lack of adaptive immune responses to HIV, either as a result of the transplant process or early treatment. In the absence of immune responses and ART, viral replication is exponential, with an $\mathrm{R}_{0}$ of 20 (20 new infected cells for each infected cell) (46). Therefore, the prolonged periods of aviremia in these patients can only be explained by the persistence of HIV in a latent, nonreplicating form. These cases provide in vivo proof for the concept of HIV latency and demonstrate the difficulty of the problem we face in trying to cure the infection.

\section{Mechanisms of persistence}

The molecular mechanisms by which latency is established and maintained have been a subject of great interest and are reviewed here by Cary et al. (34). As is described in this elegant Review, there 
are at least seven distinct mechanisms that could contribute to HIV latency. Some are clearly related to the activation state of the host cells and the fact that the transcriptional environment in resting $\mathrm{CD}^{+} \mathrm{T}$ cells is nonpermissive for $\operatorname{HIV}$ gene expression $(47,48)$. Another mechanism is related to the nature of HIV integration sites in the human genome. Following reverse transcription, the double-stranded DNA form of the HIV genome is stably integrated into host cell DNA, typically within the introns of actively expressed host genes $(49,50)$. This can lead to a phenomenon of transcription interference, in which transcription complexes initiated at the upstream host promoter interfere with HIV transcription (51).

Interestingly, the site of HIV integration may have another effect on viral persistence. Recent studies from the laboratories of Frank Maldarelli and Lisa Frenkel have used integration site analysis to identify expanded clones of cells carrying HIV proviruses $(52,53)$. The identification of multiple infected cells with exactly the same integration site in the 3 billion-base pair human genome can only be explained by the proliferation of cells after infection. This raises the disturbing possibility that latently infected cells could undergo continuous clonal expansion, hampering efforts to eliminate the reservoir. Interestingly, in some of the expanded cellular clones, the provirus is integrated in genes associated with cell survival and/or proliferation, leading to the hypothesis that the presence of the provirus in these locations alters expression of the relevant host genes in a way that promotes proliferation of the infected cells.

A major issue is whether the expanded cellular clones detected by integration site analysis harbor replication-competent virus. Detailed analysis of proviruses in resting $\mathrm{CD} 4^{+} \mathrm{T}$ cells from patients on ART has shown that the vast majority have inactivating defects in the form of hypermutation induced by apolipoprotein B mRNA editing enzyme, catalytic polypeptide-like 3G (APOBEC3G) or large internal deletions arising during reverse transcription (54). Cohn and colleagues examined 75 expanded cellular clones identified through integration site analysis and showed that all carried defective proviruses (55). Nevertheless, it remains possible that a small fraction of these expanded cellular clones harbor replication-competent HIV. Studies of the trace levels of free virus in plasma of patients on ART have previously demonstrated the dominance of clonal populations of virus, presumably derived from expanded cellular clones capable of producing virus particles (56). This issue and the overall concept of clonal expansion are discussed in detail by Frank Maldarelli in this issue (57). How the existence of expanded cellular clones alters the approach to elimination of the reservoir is a currently a subject of great interest.

\section{"Shock and kill"}

The most widely discussed approach for eliminating the reservoir is the "shock and kill" approach, in which small-molecule LRAs are used to induce HIV gene expression in the hopes that the infected cells will then die as a result of viral cytopathic effects and/or natural or induced HIV-specific immune responses (17, 58-66). This would be done in patients on ART, and it is generally assumed that reversal of latency will not result in new infection events, given the remarkable efficacy of antiretroviral drugs (67, 68). Numerous LRAs have been identified in studies with transformed cell lines carrying latent HIV proviruses and various pri- mary T cell models of latency (reviewed in refs. 69, 70). T cell activation was used to reverse latency in the original studies defining the latent reservoir $(4,5)$, but this activation is associated with substantial toxicities that preclude the use of this approach therapeutically $(71,72)$. Nevertheless, LRA activity should be compared to a positive control in which global $\mathrm{T}$ cell activation is induced by mitogens or a combination of anti-CD3 and anti-CD28 antibodies. For some of these LRAs, notably the PKC agonists, there are obvious mechanistic explanations for their activity related to effects on signaling pathways involved in $\mathrm{T}$ cell activation. For other LRAs, including the histone deacetylase (HDAC) inhibitors, the mechanisms remain controversial $(65,73)$. One of the major problems in the search for effective LRAs is that, despite impressive activity in various in vitro models, most LRAs, including the HDAC inhibitors, have weak activity in ex vivo studies using resting $\mathrm{CD}^{+}{ }^{+} \mathrm{T}$ cells from patients on ART (74). Fortunately, some combinations of LRAs are now beginning to show levels of latency reversal comparable to global $\mathrm{T}$ cell activation $(75,76)$. In clinical trials, no reduction in the reservoir has yet been demonstrated, but there is evidence for increases in cell-associated HIV RNA and for slight transient increases in plasma HIV RNA with certain HDAC inhibitors $(17,18)$. Together, these findings suggest that it will be possible to reverse HIV latency in vivo.

Reversal of latency will not reduce the reservoir unless the infected cells die as a result. Studies in primary cell models of HIV latency suggest that infected cells may not die as a result of viral cytopathic effects following LRA treatment (77). In elegant humanized mouse models of HIV infection, the elimination of infected cells has been shown to be dependent upon additional interventions designed to kill infected cells $(48,78)$. (Rapid progress in the use of humanized mice to study HIV eradication strategies is discussed by J. Victor Garcia in this issue; ref. 79.) Following latency reversal, infected cells will likely express viral antigens, but it is not clear that they will be eliminated by host CTL responses. As is discussed in a comprehensive Review by Jones and Walker in this issue (80), these responses wane in patients on ART due to the absence of antigen, and there are lingering effects of the immune exhaustion seen in untreated HIV infection $(81,82)$. In addition, recent studies have demonstrated that unless ART is started very early in the course of HIV infection, the latent reservoir is composed almost entirely of viruses with escape mutations in dominant CTL epitopes (30). Jones and Walker consider differences in features of the CTL response needed for control of active replication versus the clearance of latently infected cells and discuss new results on the targeting of epitopes, which, if mutated, lead to structural instability of the relevant viral proteins (80). Other recently identified problems with the CTL response include the negative effects of some LRAs on CTL function (84) and the possibility that infected cells may persist in anatomical sites that are inaccessible to CTLs, including the germinal centers of the lymph nodes, in which viral replication can occur in $\mathrm{CD}^{+} \mathrm{T}$ follicular helper cells $(85,86)$. Despite these problems, there is hope that an appropriately primed $\mathrm{CD}^{+} \mathrm{T}$ cell response may facilitate clearance of infected cells following latency reversal.

Recently, an unusual form of $\mathrm{T}$ cell immunity has been show to play a role in the clearance of infected cells in an important animal model of HIV infection, namely SIV infection of rhesus 
macaques. A CMV-based vaccine carrying SIV antigens provided durable protection against challenge with a pathogenic strain of SIV in $50 \%$ of vaccinated animals (87-89). In these animals, there was an initial burst of SIV replication that was rapidly controlled. The animals went on to completely clear the infection, as demonstrated by the transfer of cells to naive animals. The clearance of SIV-infected cells was mediated by $\mathrm{CD} 8^{+} \mathrm{T}$ cells, but the specificity of the cells was atypical. A broad array of SIV peptides was recognized by these cells in the context of class II and nonclassical class I MHC molecules. Whether similar noncanonical responses can be induced in humans with CMV vectors and whether they will be useful in HIV eradication strategies remain important questions.

Another approach to the elimination of infected cells involves the use of antibodies directed at the HIV envelope protein, which should be expressed on the cell surface following reversal of latency. The last few years have seen extraordinary progress in our understanding of the structure of the envelope protein and of the antibody response that it induces. In particular, there has been interest in broadly neutralizing antibodies (bNAbs) that can recognize a very wide range of HIV isolates. This work is reviewed here by Ariel Halper-Stromberg and Michel Nussenzweig (90). Nussenzweig's group developed methods for the cloning of these antibodies. Many of them have unusual structural features that are the result of a lengthy process of coevolution of the virus and the antibody response in infected individuals $(26,27)$. While these antibodies may be difficult to induce by vaccination, there is hope that passive infusion of bNAbs could contribute to the killing of latently infected cells if done in conjunction with LRA treatment. As is reviewed by Halper-Stromberg and Nussenzweig, this killing may involve the Fc receptor-dependent engagement of NK cells (90).

\section{Summary}

The renewed interest in HIV cure has brought together diverse lines of HIV research, including studies of HIV molecular biology, pathogenesis, and vaccine development, all of which may end up contributing to a cure. Although infected individuals with access to ART can now expect much better clinical outcomes than those in the pre-ART era, they still face a lifetime of treatment. The global burden of treating every infected individual for life with combinations of expensive antiretroviral drugs is a strong motivation for finding simple, scalable, curative regimens that can reduce the viral reservoir and allow prolonged ART-free remissions.

\section{Acknowledgments}

This work was supported by the Martin Delaney Collaboratory of AIDS Researchers for Eradication and Delaney AIDS Research Enterprise (NIH grants AI096113 and 1U19AI096109), by an amfAR Research Consortium on HIV Eradication collaborative research grant from the Foundation for AIDS Research (amfAR 108165-50-RGRL), by the Johns Hopkins Center for AIDS Research (P30AI094189), by NIH grant 43222, by the Howard Hughes Medical Institute, and by the Bill and Melinda Gates Foundation.

Address correspondence to: Robert F. Siliciano, Room 879, Edward D. Miller Research Building, 733 N. Broadway, Baltimore, Maryland 21205, USA. Phone: 410.955.2958; E-mail: rsiliciano@jhmi.edu.
1. Hammer SM, et al. A controlled trial of two nucleoside analogues plus indinavir in persons with human immunodeficiency virus infection and CD4 cell counts of 200 per cubic millimeter or less. AIDS Clinical Trials Group 320 Study Team. N Engl J Med. 1997;337(11):725-733.

2. Gulick RM, et al. Treatment with indinavir, zidovudine, and lamivudine in adults with human immunodeficiency virus infection and prior antiretroviral therapy. $N$ Engl J Med. 1997;337(11):734-739.

3. Perelson AS, et al. Decay characteristics of HIV-1infected compartments during combination therapy. Nature. 1997;387(6629):188-191.

4. Chun TW, Finzi D, Margolick J, Chadwick K, Schwartz D, Siliciano RF. In vivo fate of HIV-1-infected T cells: quantitative analysis of the transition to stable latency. Nat Med.1995;1(12):1284-1290.

5. Chun TW, et al. Quantification of latent tissue reservoirs and total body viral load in HIV-1 infection. Nature. 1997;387(6629):183-188.

6. Finzi D, et al. Identification of a reservoir for HIV-1 in patients on highly active antiretroviral therapy. Science. 1997;278(5341):1295-1300.

7. Wong JK, et al. Recovery of replication-competent HIV despite prolonged suppression of plasma viremia. Science. 1997;278(5341):1291-1295.

8. Chun TW, et al. Presence of an inducible HIV-1 latent reservoir during highly active antiretroviral therapy. Proc Natl Acad Sci U S A. 1997;94(24):13193-13197.

9. Finzi D, et al. Latent infection of $\mathrm{CD} 4^{+} \mathrm{T}$ cells provides a mechanism for lifelong persistence of
HIV-1, even in patients on effective combination therapy. Nat Med.1999;5(5):512-517.

10. Siliciano JD, et al. Long-term follow-up studies confirm the stability of the latent reservoir for HIV-1 in resting CD4 ${ }^{+} \mathrm{T}$ cells. Nat Med. 2003;9(6):727-728.

11. Strain MC, et al. Heterogeneous clearance rates of long-lived lymphocytes infected with HIV: intrinsic stability predicts lifelong persistence. Proc Natl Acad Sci US A. 2003;100(8):4819-4824.

12. Gunthard HF, et al. Antiretroviral treatment of adult HIV infection: 2014 recommendations of the International Antiviral Society-USA Panel. JAMA. 2014;312(4):410-425.

13. van Sighem AI, Gras LA, Reiss P, Brinkman K, de Wolf F, ATHENA national observational cohort study. Life expectancy of recently diagnosed asymptomatic HIV-infected patients approaches that of uninfected individuals. AIDS. 2010;24(10):1527-1535.

14. Mills EJ, et al. Life expectancy of persons receiving combination antiretroviral therapy in low-income countries: a cohort analysis from Uganda. Ann Intern Med. 2011;155(4):209-216.

15. Nakagawa F, May M, Phillips A. Life expectancy living with HIV: recent estimates and future implications. Curr Opin Infect Dis. 2013;26(1):17-25.

16. Johnson LF, et al. Life expectancies of South African adults starting antiretroviral treatment: collaborative analysis of cohort studies. PLoS Med. 2013;10(4):e1001418.

17. Archin NM, et al. Administration of vorinostat disrupts HIV-1 latency in patients on antiretrovi- ral therapy. Nature. 2012;487(7408):482-485.

18. Sogaard OS, et al. The depsipeptide romidepsin reverses HIV-1 latency in vivo. PLoS Pathog. 2015;11(9):e1005142.

19. Hutter G, et al. Long-term control of HIV by CCR5 $\Delta 32 / \Delta 32$ stem-cell transplantation. $N$ Engl JMed. 2009;360(7):692-698.

20. Yukl SA, et al. Challenges in detecting HIV persistence during potentially curative interventions: a study of the Berlin patient. PLoS Pathog. 2013;9(5):e1003347.

21. Massanella M, Richman DD. Measuring the latent reservoir in vivo. J Clin Invest. 2016;126(2):464-472.

22. Crooks AM, et al. Precise quantitation of the latent HIV-1 reservoir: implications for eradication strategies. J Infect Dis. 2015;212(9):1361-1365.

23. Perng GC, Jones C. Towards an understanding of the herpes simplex virus type 1 latency-reactivation cycle. Interdiscip Perspect Infect Dis. 2010;2010:262415.

24. Piatak M Jr, et al. High levels of HIV-1 in plasma during all stages of infection determined by competitive PCR. Science. 1993;259(5102):1749-1754.

25. Borrow P, et al. Antiviral pressure exerted by HIV-1-specific cytotoxic T lymphocytes (CTLs) during primary infection demonstrated by rapid selection of CTL escape virus. Nat Med. 1997;3(2):205-211.

26. Wei X, et al. Antibody neutralization and escape by HIV-1. Nature. 2003;422(6929):307-312.

27. Richman DD, Wrin T, Little SJ, Petropoulos CJ. Rapid evolution of the neutralizing antibody 
response to HIV type 1 infection. Proc Natl Acad Sci U S A. 2003;100(7):4144-4149.

28. Jones NA, et al. Determinants of human immunodeficiency virus type 1 escape from the primary $\mathrm{CD}^{+}$cytotoxic $\mathrm{T}$ lymphocyte response. J Exp Med. 2004;200(10):1243-1256.

29. Wood N, et al. HIV evolution in early infection: selection pressures, patterns of insertion and deletion, and the impact of APOBEC. PLoS Pathog. 2009;5(5):e1000414.

30. Deng K, et al. Broad CTL response is required to clear latent HIV-1 due to dominance of escape mutations. Nature. 2015;517(7534):381-385.

31. Razooky BS, Pai A, Aull K, Rouzine IM, Weinberger LS. A hardwired HIV latency program. Cell. 2015;160(5):990-1001.

32. Rouzine IM, Weinberger AD, Weinberger LS. An evolutionary role for HIV latency in enhancing viral transmission. Cell. 2015;160(5):1002-1012.

33. Bleul CC, Wu L, Hoxie JA, Springer TA, Mackay CR. The HIV coreceptors CXCR4 and CCR5 are differentially expressed and regulated on human T lymphocytes. Proc Natl Acad Sci U S A. 1997;94(5):1925-1930.

34. Cary DC, Fujinaga K, Peterlin BM. Molecular mechanisms of HIV latency. J Clin Invest. 2016;126(2):448-454.

35. Ho DD, Neumann AU, Perelson AS, Chen W, Leonard JM, Markowitz M. Rapid turnover of plasma virions and CD4 lymphocytes in HIV-1 infection. Nature. 1995;373(6510):123-126.

36. Wei X, et al. Viral dynamics in human immunodeficiency virus type 1 infection. Nature. 1995;373(6510):117-122.

37. Descours B, et al. SAMHD1 restricts HIV-1 reverse transcription in quiescent CD4(+) T-cells. Retrovirology. 2012;9:87-4690.

38. Baldauf HM, et al. SAMHD1 restricts HIV-1 infection in resting CD4(+) T cells. Nat Med. 2012;18(11):1682-1687.

39. Doitsh G, et al. Cell death by pyroptosis drives CD4 T-cell depletion in HIV-1 infection. Nature. 2014;505(7484):509-514.

40. Kuritzkes DR. Hematopoietic stem cell transplantation for HIV cure. J Clin Invest. 2016;126(2):432-437.

41. Henrich TJ, et al. Long-term reduction in peripheral blood HIV type 1 reservoirs following reduced-intensity conditioning allogeneic stem cell transplantation. J Infect Dis. 2013;207(11):1694-1702.

42. Henrich TJ, et al. Antiretroviral-free HIV-1 remission and viral rebound after allogeneic stem cell transplantation: report of 2 cases. Ann Intern Med. 2014;161(5):319-327.

43. Davey RT Jr, et al. HIV-1 and T cell dynamics after interruption of highly active antiretroviral therapy (HAART) in patients with a history of sustained viral suppression. Proc Natl Acad Sci U S A. 1999;96(26):15109-15114.

44. Rothenberger MK, et al. Large number of rebounding/founder HIV variants emerge from multifocal infection in lymphatic tissues after treatment interruption. Proc Natl Acad Sci U S A. 2015;112(10):E1126-E1134.

45. Persaud D, et al. Absence of detectable HIV-1 viremia after treatment cessation in an infant. $N$ Engl J Med. 2013;369(19):1828-1835.
46. Little SJ, McLean AR, Spina CA, Richman DD, Havlir DV. Viral dynamics of acute HIV-1 infection. J Exp Med. 1999;190(6):841-850.

47. Siekevitz M, Josephs SF, Dukovich M, Peffer N, Wong-Staal F, Greene WC. Activation of the HIV-1 LTR by T cell mitogens and the trans-activator protein of HTLV-I. Science. 1987;238(4833):1575-1578.

48. Brooks DG, Arlen PA, Gao L, Kitchen CM, Zack JA. Identification of T cell-signaling pathways that stimulate latent HIV in primary cells. Proc Natl Acad Sci U S A. 2003;100(22):12955-12960.

49. Schroder AR, Shinn P, Chen H, Berry C, Ecker JR, Bushman F. HIV-1 integration in the human genome favors active genes and local hotspots. Cell. 2002;110(4):521-529.

50. Han Y, et al. Resting CD $4^{+} \mathrm{T}$ cells from human immunodeficiency virus type 1 (HIV-1)-infected individuals carry integrated HIV-1 genomes within actively transcribed host genes. J Virol. 2004;78(12):6122-6133.

51. Lenasi T, Contreras X, Peterlin BM. Transcriptional interference antagonizes proviral gene expression to promote HIV latency. Cell Host Microbe. 2008;4(2):123-133.

52. Maldarelli F, et al. HIV latency. Specific HIV integration sites are linked to clonal expansion and persistence of infected cells. Science. 2014;345(6193):179-183.

53. Wagner TA, et al. HIV latency. Proliferation of cells with HIV integrated into cancer genes contributes to persistent infection. Science. 2014;345(6196):570-573.

54. Ho YC, et al. Replication-competent noninduced proviruses in the latent reservoir increase barrier to HIV-1 cure. Cell. 2013;155(3):540-551.

55. Cohn LB, et al. HIV-1 integration landscape during latent and active infection. Cell. 2015;160(3):420-432.

56. Bailey JR, et al. Residual human immunodeficiency virus type 1 viremia in some patients on antiretroviral therapy is dominated by a small number of invariant clones rarely found in circulating $\mathrm{CD} 4^{+}$ T cells. JVirol. 2006;80(13):6441-6457.

57. Maldarelli F. The role of HIV integration in viral persistence: no more whistling past the proviral graveyard. JClin Invest. 2016;126(2):438-447.

58. Richman DD, Margolis DM, Delaney M, Greene WC, Hazuda D, Pomerantz RJ. The challenge of finding a cure for HIV infection. Science. 2009;323(5919):1304-1307.

59. Korin YD, Brooks DG, Brown S, Korotzer A, Zack JA. Effects of prostratin on T-cell activation and human immunodeficiency virus latency. J Virol. 2002;76(16):8118-8123.

60. Scripture-Adams DD, Brooks DG, Korin YD, Zack JA. Interleukin-7 induces expression of latent human immunodeficiency virus type 1 with minimal effects on T-cell phenotype. J Virol. 2002;76(24):13077-13082.

61. Brooks DG, et al. Molecular characterization, reactivation, and depletion of latent HIV. Immunity. 2003;19(3):413-423.

62. He G, Ylisastigui L, Margolis DM. The regulation of HIV-1 gene expression: the emerging role of chromatin. DNA Cell Biol. 2002;21(10):697-705.

63. Lehrman G, Ylisastigui L, Bosch RJ, Margolis DM. Interleukin-7 induces HIV type 1 outgrowth from peripheral resting $\mathrm{CD}^{+} \mathrm{T}$ cells. J Acquir Immune Defic Syndr. 2004;36(5):1103-1104.

64. Ylisastigui L, Archin NM, Lehrman G, Bosch RJ, Margolis DM. Coaxing HIV-1 from resting CD4 T cells: histone deacetylase inhibition allows latent viral expression. AIDS. 2004;18(8):1101-1108.

65. Archin NM, Espeseth A, Parker D, Cheema M, Hazuda D, Margolis DM. Expression of latent HIV induced by the potent HDAC inhibitor suberoylanilide hydroxamic acid. AIDS Res Hum Retroviruses. 2009;25(2):207-212.

66. The International AIDS Society Scientific Working Group on HIV Cure, et al. Towards an HIV cure: a global scientific strategy. Nat Rev Immunol. 2012;12(8):607-614.

67. Shen L, et al. Dose-response curve slope sets class-specific limits on inhibitory potential of anti-HIV drugs. Nat Med. 2008;14(7):762-766

68. Jilek BL, et al. A quantitative basis for antiretroviral therapy for HIV-1 infection. Nat Med. 2012;18(3):446-451.

69. Spina CA, et al. An in-depth comparison of latent HIV-1 reactivation in multiple cell model systems and resting $\mathrm{CD}^{+} \mathrm{T}$ cells from aviremic patients. PLoS Pathog. 2013;9(12):e1003834.

70. Xing S, Siliciano RF. Targeting HIV latency: pharmacologic strategies toward eradication. Drug Discov Today. 2013;18(11-12):541-551.

71. Chatenoud L, et al. In vivo cell activation following OKT3 administration. Systemic cytokine release and modulation by corticosteroids. Transplantation. 1990;49(4):697-702.

72. Prins JM, et al. Immuno-activation with anti-CD3 and recombinant human IL-2 in HIV-1-infected patients on potent antiretroviral therapy. AIDS. 1999;13(17):2405-2410.

73. Bartholomeeusen K, Fujinaga K, Xiang Y, Peterlin BM. Histone deacetylase inhibitors (HDACis) that release the positive transcription elongation factor $\mathrm{b}(\mathrm{P}-\mathrm{TEFb})$ from its inhibitory complex also activate HIV transcription. J Biol Chem. 2013;288(20):14400-14407.

74. Bullen CK, Laird GM, Durand CM, Siliciano JD, Siliciano RF. New ex vivo approaches distinguish effective ineffective single agents for reversing HIV-1 latency in vivo. Nat Med. 2014;20(4):425-429.

75. Laird GM, et al. Ex vivo analysis identifies effective HIV-1 latency-reversing drug combinations. J Clin Invest. 2015;125(5):1901-1912.

76. Darcis G, et al. An in-depth comparison of latency-reversing agent combinations in various in vitro and ex vivo HIV-1 latency models identified Bryostatin-1+JQ1 and Ingenol-B+JQ1 to potently reactivate viral gene expression. PLoS Pathog. 2015;11(7):e1005063.

77. Shan L, et al. Stimulation of HIV-1-specific cytolytic T lymphocytes facilitates elimination of latent viral reservoir after virus reactivation. Immunity. 2012;36(3):491-501.

78. Denton PW, et al. Targeted cytotoxic therapy kills persisting HIV infected cells during ART. PLOS Pathog. 2014;10(1):e1003872.

79. Garcia JV. In vivo platforms for analysis of HIV persistence and eradication. JClin Invest. 2016;126(2):424-431.

80. Jones RB, Walker BD. HIV-specific CD8 ${ }^{+}$ T cells and HIV eradication. J Clin Invest. 2016;126(2):455-463. 
81. Day CL, et al. PD-1 expression on HIV-specific $\mathrm{T}$ cells is associated with T-cell exhaustion and disease progression. Nature. 2006;443(7109):350-354

82. Kaufmann DE, Walker BD. PD-1 and CTLA-4 inhibitory cosignaling pathways in HIV infection and the potential for therapeutic intervention. JImmunol. 2009;182(10):5891-5897.

83. Pereyra F, et al. HIV control is mediated in part by $\mathrm{CD}^{+} \mathrm{T}$-cell targeting of specific epitopes. JVirol. 2014;88(22):12937-12948.

84. Jones RB, et al. Histone deacetylase inhibitors impair the elimination of HIV-infected cells by cytotoxic T-lymphocytes. PLoS Pathog. 2014;10(8):e1004287.

85. Perreau M, et al. Follicular helper T cells serve as the major CD4 $\mathrm{T}$ cell compartment for HIV-1 infection, replication, and production. J Exp Med. 2013;210(1):143-156.

86. Fukazawa Y, et al. B cell follicle sanctuary permits persistent productive simian immunodeficiency virus infection in elite controllers. Nat Med. 2015;21(2):132-139.

87. Hansen SG, et al. Profound early control of highly pathogenic SIV by an effector memory T-cell vaccine. Nature. 2011;473(7348):523-527.

88. Hansen SG, et al. Immune clearance of highly pathogenic SIV infection. Nature. 2013;502(7469):100-104.

89. Hansen SG, et al. Cytomegalovirus vectors violate $\mathrm{CD}^{+} \mathrm{T}$ cell epitope recognition paradigms. Science. 2013;340(6135):1237874.

90. Halper-Stromberg A, Nussenzweig MC. Towards HIV-1 remission: potential roles for broadly neutralizing antibodies. JClin Invest. 2016;126(2):415-423. 